\title{
Defects in graphene help perovskite dots grow
}

Sci. Adv. 6, eaay5225 (2020)

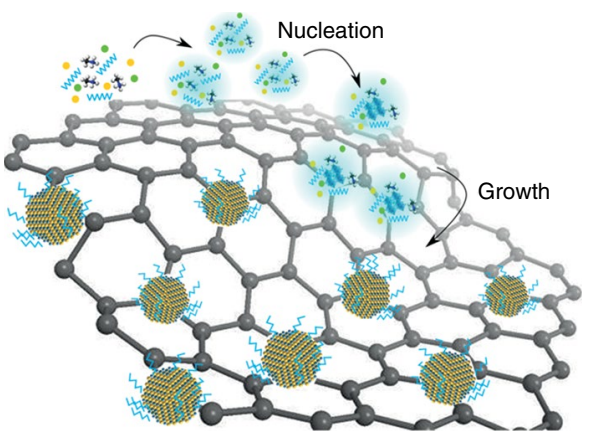

Credit: AAAS

Graphene is a promising material with which to build optoelectronic devices due to its high mobility. Photodetectors based on graphene, however, typically have low responsivity because of the limited light absorption of the material. One approach to improve the responsivity is to integrate perovskite quantum dots, which have better light absorption than graphene. Tania Roy, Jayan Thomas and colleagues at the University of Central Florida have now developed a method of growing methylammonium lead bromide perovskite quantum dots directly from a single layer of graphene.

The technique creates graphene/quantum dot superstructures using a defect-mediated process in which a perovskite precursor is added to graphene. In particular, perovskite dots tend to form at defect sites in graphene, where the surface energy promotes the formation of a crystal. Using the superstructures, the researchers created phototransistors on silicon with a recordhigh responsivity of $1.4 \times 10^{8}$ amps per watt and a detectivity of $4.72 \times 10^{15}$ Jones. The superstructures could also potentially function as gate-tuneable photonic synapses. For example, with the help of simulations, Roy, Thomas and colleagues show that these artificial synapses could be used to build a spiking neural network that can perform unsupervised machine learning and face recognition.

Christiana Varnava

Published online: 20 March 2020

https://doi.org/10.1038/s41928-020-0392-1 